SciDioc

International Journal of Dentistry and Oral Science (IJDOS)

ISSN: 2377-8075

\title{
Does the Number of Attempts of Endotracheal Intubation Influence the Incidence of Post-Extuba- tion Dysphagia in Patients Undergoing Maxillofacial Surgical Procedures? - A Prospective Analysis
}

Research Article

Rajesh P ${ }^{1}$, Prakyatha Brasanna Shetty², Vaishali V²

${ }^{1}$ Professor and Head, Department of Oral and Maxillofacial Surgery, Chettinad Dental College and Research Institute, India.

${ }_{2}^{2}$ Post Graduate, Department of Oral and Maxillofacial Surgery, Chettinad Dental College and Research Institute, India.

Abstract

Aim: To evaluate the incidence and association of post-extubation dysphagia (PED) with varying number of attempts of endotracheal intubation in patients undergoing maxillofacial surgical procedures.

Settings: One of the unnoticed complications of endotracheal intubation, yet with tragic consequences is post-extubation dysphagia. In a post maxillofacial surgical patient, this is masked by the extensiveness of the surgery and the immobilization protocols and hence need to be attended with utmost care.

Materials and Methods: A two-year prospective analysis of patients undergoing maxillofacial surgical procedures for varied cause was done. Data regarding age, gender, medical history, indication for surgery, pre-anaesthetic evaluation, intraoperative records related to the number of intubation attempts and any complications associated and a water swallow test to test for PED 24 hours after surgery were recorded and subjected to statistical analysis.

Results: Mean age of the population was 35.1 years and was predominantly male. About $62.5 \%$ of them were intubated by fiberoptic intubation, $35 \%$ by naso-endotracheal and $5 \%$ by oro-endotracheal intubation. $65 \%$ of them were intubated more than once and a maximum of 7 attempts was done to secure the airway. 19 patients had PED and it was significantly associated with the number of attempts of intubation with $\mathrm{p}<0.001$.

Conclusion: PED is the tip of the iceberg and could indicate a serious underlying complication that could result in fatal complications if neglected and thus alarms the surgeon to watch out closely and address accordingly.

Keywords: Dysphagia; Maxillofacial Surgery; Endotracheal Intubation; Airway.

\section{Introduction}

Endotracheal intubation is the choice of securing the airway during surgical procedures under general anaesthesia. Intubation in patients indicated for oral and maxillofacial surgical procedures are quite challenging and different from others. Often difficult intubation is anticipated with increased extensiveness of the injuries or pathology and failure of intubation in the first attempt is not so uncommon. But the post extubation complications associated as a result has to be attended with care to avoid flare-ups of unnecessary consequences. One such complication is post extubation dysphagia especially in maxillofacial surgical patients which in combination with post-surgical immobilization of the jaw keeps any serious complications like aspiration pneumonia latent. This study aims to throw some light on the importance of addressing the dysphagia in post-surgical patient and to evaluate its incidence with difficulty in intubation.

\section{Materials and Methods}

A prospective analysis of 40 patients who reported to the outpatient department, and emergency department of our institution from November 2017 to November 2019 seeking surgical management of maxillofacial injuries, pathologies and orthognathic surgeries under general anaesthesia. Ethical clearance was obtained from the Institutional Human Ethics Committee and informed consent was obtained from the patients before the surgery. Patients indicated for Open reduction and internal fixation

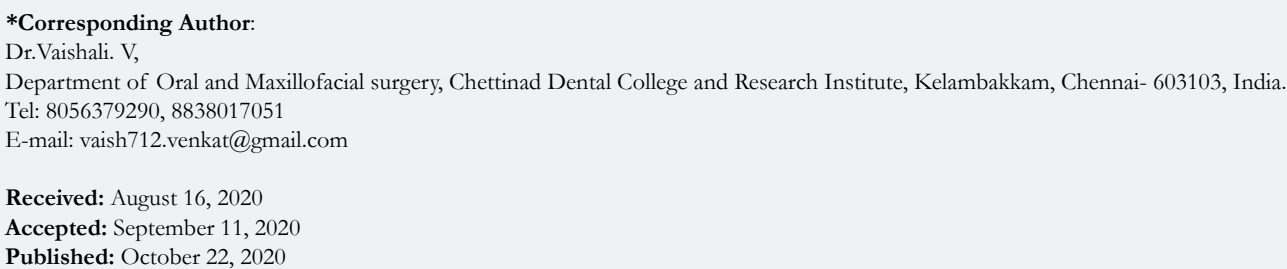

Citation: Rajesh P, Prakyatha Brasanna Shetty, Vaishali V. Does the Number of Attempts of Endotracheal Intubation Influence the Incidence of Post-Extubation Dysphagia in Patients Undergoing Maxillofacial Surgical Procedures? - A Prospective Analysis. Int J Dentistry Oral Sci. 2020;7(10):860-863. doi: http://dx.doi.org/10.19070/2377-8075-20000170

Copyright: Vaishali $V^{\top} 2020$. This is an open-access article distributed under the terms of the Creative Commons Attribution License, which permits unrestricted use, distribution and reproduction in any medium, provided the original author and source are credited. 
of various maxillo-mandibular fractures, space infections, surgical removal of pathologies like cyst enucleation, tumour resection, cleft repair and removal of temporomandibular joint ankylosis and those requiring jaw correction surgeries i.e. orthognathic surgeries were included in the study. Those patients falling under the categories ASA III and ASA IV, those with surgical airway and known cases of obstructive and restrictive lung disorders were excluded from the study. After obtaining informed consent, demographic details, reason for the indication of surgery, complete pre-anaesthetic evaluation, intraoperative records of the type of intubation chosen and the number of attempts for successful intubation, any other intraoperative complications associated and incidence of post extubation dysphagia that was recorded with water swallowing test 24 hours after the surgery. The endotracheal intubation was performed by the experienced and skilled anaesthesiologist of our institution. All the data were recorded and subjected to statistical analysis.

\section{Statistical analysis}

The collected data were analysed with IBM.SPSS statistics software 21.0 Version. Frequency distribution was calculated for categorical. Descriptive statistics were calculated for numerical data. Independent $t$ test was used to find the significance and correlation between variables. In all the above statistical tools the probability value .05 is considered as significant level.

\section{Results}

A total of 40 patients were enrolled in the study. About $82.5 \%$ $(n=33)$ of them were males and $17.5 \%(n=7)$ were females. Mean age of the population was 35.1 years. Figure 1 depicts the fre- quency of the type of intubation chosen.

Fiber-optic intubation was the highest used with $62.5 \%(n=25)$, followed by direct nasal intubation in 32.5\% ( $n=13)$ and finally orotracheal intubation in $5 \%(n=2)$. Table 1 shows the number of attempts used for successful intubation. About 35\% ( $n=14)$ of the population was intubated successfully in the first attempt, $22.5 \%(n=9)$ in their second attempt, $15 \%(n=6)$ in the fourth attempt and $10 \%(n=4)$ in the third attempts and rest of them had five attempts and more for successful intubation.

$47.5 \%(n=19)$ of them were found to have post extubation dysphagia when screened 24 hours after surgery. When the number of intubation attempts and incidence of post-extubation dysphagia was correlated, there existed a positive correlation between both, and it was statistically significant with $\mathrm{p}<0.001$.

\section{Discussion}

Securing the airway during maxillofacial procedures is peculiar from others due to the shared regional anatomy of both. It is because the maxillofacial injuries have direct impact on the respiratory tract thereby injuring it as in case of trauma or indirectly restricting the access due to limited mouth opening or obstructive nature of the pathology or the tissue injury. This poses a challenge to the anaesthesiologist to carry out detailed examination of the upper respiratory tract to opt for the indicated method of securing the airway. Also, they are not left with the freedom of manipulation and mobilization of the maxillofacial structures during examination or intubation due to the extensiveness of the injury or pathology and due to immobilization protocols to be followed based on the surgical plan. Prior to the surgical procedure, a thor-

Figure 1. Types of Endotracheal intubation employed.

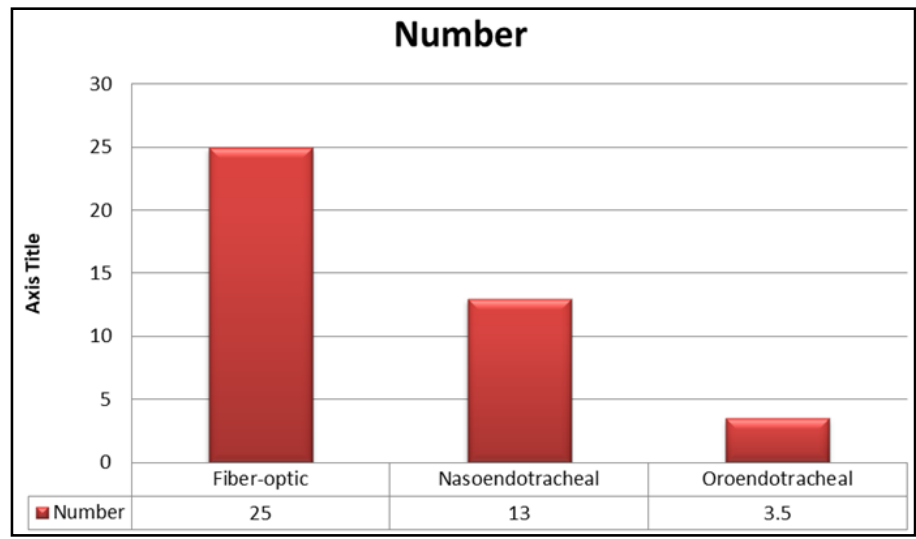

Table 1. Number of attempts of intubation.

\begin{tabular}{|c|c|c|}
\hline Attempt & Frequency & Percent \\
\hline 1 & 14 & 35 \\
\hline 2 & 9 & 22.5 \\
\hline 3 & 4 & 10 \\
\hline 4 & 6 & 15 \\
\hline 5 & 4 & 10 \\
\hline 6 & 1 & 2.5 \\
\hline 7 & 2 & 5 \\
\hline total & 40 & 100 \\
\hline
\end{tabular}


ough evaluation of the patient is mandatory that includes LEMON assessment - external predictors of the difficult airway that measures the length of the neck, thyromental distance, atlantoaxial mobility, Mouth opening, Mallampati classification, and any obstructed airway seen as stridor and neck mobility [1]. In cases of maxillofacial trauma, the extent of the fracture, mobile bony segments, the elevated floor of the mouth, soft tissue edema, and loose teeth with the risk of aspiration, CSF leak, limited mobility due to muscle spasm and, in case of any pathology, its extent, swelling or ulceration, maximum mouth opening, obstructed airway should be noted and based on the evaluation difficulty of intubation should be anticipated. Thus the choice of intubation in maxillofacial surgical procedures is always a team approach and involves various factors that determine the success of intubation.

Every method of intubation has its indications and contraindications. However endotracheal intubation either nasal or oral way, remains the standard way of securing the airway [2]. But limited mouth opening in maxillofacial surgical patient disables direct visualization of vocal cords which is a pre-requisite for successful endotracheal intubation. In such cases, awake fiber-optic intubation is preferred that enables the anaesthesiologist to visualize the vocal cords indirectly. The disadvantage that entails this method is obscuring of vision by salivary secretions and blood but the failure of intubation is minimal than the other direct techniques and especially when the mouth opening is compromised. Method of intubation and its success has important clinical implications. Complications that results from the endotracheal intubation could be major or minor [3]. Major complications include granulomas, laryngeal ulceration or vocal cord injury including paralysis while minor complications include sore throat, laryngeal edema, stridor and dysphagia. The severity of complications depends on the selection of the tube, proper instrumentation and placement of the tube, number of attempts for successful intubation, duration of intubation, pre-existing injuries to the airway and extubation related. One important complication that often goes unnoticed is the post extubation dysphagia that indicates more serious systemic issues in a post-operative patient [4].

Post extubation dysphagia is defined as the inability or difficulty to effectively and safely transfer food and liquid from the mouth to the stomach after extubation. Clinically it is defined as the inability to drink $50 \mathrm{ml}$ of water within 48 hours of extubation. Leder et al reported a higher incidence of PED in trauma and critically ill patients intubated endotracheally for mechanical ventilation [5]. Multifactorial causation of PED has been described including mechanical causes, cognitive disturbances and residual effects of the drugs. Mechanical injury due to improper size of the tube and prolonged intubation causes mucosal inflammation leading to loss of architecture, atrophy of the muscles if prolonged, reduced proprioception and reduced laryngeal circulation. Any dysregulation in the swallowing reflexes due to neuromuscular disorders or cognitive impairment due to traumatic brain injury significantly contributes to PED. Those with neuromuscular disorders, low Glasgow coma scores, severely injured, old age, prolonged ventilation, forced supine position, head and neck pathologies, placement of nasogastric tube adds risk to developing PED. Bordon et al reported from his study in trauma patients that with every added day of intubation of the patient, $14 \%$ rise in the risk of incidence of PED and patients who were 55 years of age and above had $37 \%$ increased risk. Macht et al., [6] proposed six mechanisms to be etiological for PED in post-extubation patients:
1) Direct trauma to the anatomy of the throat (vocal cords, tongue base, epiglottis, arytenoids by endotracheal or tracheostomy tubes 2) Muscular weakness due to nerve and muscle damage (disuse atrophy; critical illness neuropathy and myopathy

3) Loss of normal sensation in the oropharynx and larynx

4) Impaired sensorium generally (delirium, sedation)

5) Gastroesophageal reflux

6) Out-of-sync breathing and swallowing in people with tachypnea before and/or after extubation.

Undiagnosed dysphagia in post-extubation patients leads to bronchoaspiration and related complications like pneumonia, malnutrition, increased hospital stay and thereby interferes the recovery period of the patient. Sassi et al reported that $50 \%$ of the patients with difficult or prolonged intubation that presented with postextubation dysphagia developed broncho-aspiration7. Such scenario gets worse in a maxillofacial patient due to the overt influence of other factors like static immobilized jaw, pain and swelling with alternative route of nutrition that narrows the focus of the patient and the surgeons during the first few days after surgery. Meanwhile in rare occasions, if the respiratory and pharyngeal complications are not treated results in catastrophic complications leading to morbidities and mortalitites.

A number of methods are in use to diagnose PED [7]. Commonly used method is the bedside evaluation (BSE) by a speech pathologist. It is a multifaceted test comprising of an interview, clinical assessment of respiratory tract and functional changes if any. Another most often employed screening test for dysphagia is the water swallow test [8]. When the severity of the condition has to be graded, various other instrumental tests are preferred. Video fluoroscopic swallow study (VFSS), Fiberoptic endoscopic evaluation of swallowing, Ultrasonography, $\mathrm{pH}$ manometry and scintigraphy. Of these VFSS and FEES are considered to be the gold standard for diagnosis of PED as they portray a real time image of various deglutition stages which aids in the accurate diagnosis and prompt management of the condition [3]. In maxillofacial surgical patients it is mandatory to check for the incidence of PED post-surgically. In many instances PED is either unnoticed due to the extensiveness of the surgery or masked by the jaw immobilization protocols. The same reason calls for alternative methods of nutrition to not interfere in the healing of the surgical wound. By the time the patient reverts to oral intake, any minor injuries to the pharynx would have healed and thus passes unseen. This study intended to uncover the latent threat of the PED, even if rare, and highlight the importance of thorough examination of the patient during the post surgical period to watch out for any signs and symptoms of PED though could be indicating an ongoing aspiration pneumonia or related complications.

In our study, the mean age group of the population was 35.1 years and the gender was predominantly male. This relieves the influence of age on the incidence of dysphagia thereby eliminating the confounding bias. Fiber-optic intubation was the frequently chosen method of intubation (62.5\%) followed by direct nasoendotracheal intubation $(35 \%)$ and finally by the oro-endotracheal intubation. This differs from the results of the Sarasvat et al., and Rashiuddin et al., where direct nasal intubation was the preferred choice of intubation [9]. But this could be because those studies involved only maxillofacial trauma while in our study patients undergoing all types of maxillofacial surgical procedures were 
involved. Also the demographics, extensiveness of the etiology, anticipated difficulty in airway and the anaesthesiologists' preference has a major role in the choice of airway. About $65 \%$ of the patients were not intubated successfully in the first attempt. $22 \%$ had to be intubated twice, $15 \%$ four times and $10 \%$ three times and the rest multiple times for a successful intubation. This could be attributed again to the complexity of the injuries and their obscurity to the evaluation. Significant proportion of the lot developed PED (47.5\%) and it had a strong positive correlation with the number of failed attempts of intubation. Thus difficult intubation could indicate potential complication of PED in the post-surgical period.

Thus it is expected of every maxillofacial surgeon to express a high index of suspicion during their evaluation for the symptoms of PED to avoid unlikely complications. Knowledge of the attributes of difficult airway, skills of managing the same, accurate recognition of a failed airway is important while securing the airway [10].

\section{Conclusion}

Post extubation dysphagia is not uncommon even in patients without pre-existing pathologic states. Owing to the life threatening consequences that could occur if PED is neglected, every maxillofacial surgeon to monitor the patient closely. The risk of dysphagia escalates with increased attempts of endotracheal intubation and it should alarm the surgeon to anticipate PED and watch out for the same to effectively manage the condition.

\section{Refereces}

[1]. Raval CB, Rashiduddin M. Airway management in patients with maxillofacial trauma - A retrospective study of 177 cases. Saudi J Anaesth. 2011; 5(1): 9-14. PMID: 21655009.

[2]. Barak M, Bahouth H, Leiser Y, Abu El-Naaj I. Airway Management of the Patient with Maxillofacial Trauma: Review of the Literature and Suggested Clinical Approach. Biomed Res Int. 2015; 2015: 724032. PMID: 26161411.

[3]. Tay JYY, Tan WKS, Chen FG, Koh KF, Ho V. Postoperative sore throat after routine oral surgery: influence of the presence of a pharyngeal pack. British Journal of Oral and Maxillofacial Surgery. 2002; 40(1): 60-63.

[4]. Rassameehiran S, Klomjit S, Mankongpaisarnrung C, Rakvit A. Postextubation Dysphagia. Proc (Bayl Univ Med Cent). 2015 Jan; 28(1):18-20. PMID: 25552788.

[5]. Leder SB, Cohn SM, Moller BA. Fiberoptic endoscopic documentation of the high incidence of aspiration following extubation in critically ill trauma patients. Dysphagia. 1998; 13(4): 208-212. PMID: 9716751.

[6]. Macht M, Wimbish T, Clark BJ, Benson AB, Burnham EL, Williams A, et al. Postextubation dysphagia is persistent and associated with poor outcomes in survivors of critical illness. Crit Care. 2011; 15(5): R231. PMID: 21958475.

[7]. Medeiros Gisele Chagas de, Sassi Fernanda Chiarion, Mangilli Laura Davison, Zilberstein Bruno, Andrade Claudia Regina Furquim de. Clinical dysphagia risk predictors after prolonged orotracheal intubation. Clinics (Sao Paulo). 2014; 69( 1 ): 8-14. PMID: 24473554.

[8]. Tsai MH, Ku SC, Wang TG, Hsiao TY, Lee JJ, Chan DC, et al. Swallowing dysfunction following endotracheal intubation: Age matters. Medicine (Baltimore). 2016; 95(24): e3871. PMID: 27310972.

[9]. Saraswat V. Airway management in Maxillofacial trauma: A Restrospective Review of 127 cases. Indian J Anaesth. 2008; 52: 311-6.

[10]. Skoretz SA, Flowers HL, Martino R. The Incidence of Dysphagia Following Endotracheal Intubation. Chest. 2010; 137(3): 665-673. PMID: 20202948. 